\title{
Surface phase transitions in two-component systems
}

\author{
Giorgio Mazzeo $^{\text {a, }}{ }^{\text {, Enrico Carlon }}{ }^{\mathrm{b}}$, Henk van Beijeren ${ }^{\mathrm{b}}$ \\ a Dipartimento di Fisica, Università di Genova, and INFM, via Dodecaneso 33, 16146 Genova, Italy \\ b Instituut voor Theoretische Fysica, Universiteit Utrecht, Postbus 80006, 3508 TA Utrecht, The Netherlands
}

Received 5 September 1995; accepted for publication 31 October 1995

\begin{abstract}
The (001) surface of a two-component crystal of $\mathrm{CsCl}$ structure is described by a six vertex model with staggered vertex weights. Its rich phase diagram includes a $c(2 \times 2)$ reconstructed phase undergoing, when temperature is increased, a second order phase transition into a disordered flat phase. This is followed, in a certain range of the parameters' values, by two Kosterlitz-Thouless transitions, the first one into a rough phase, and the second one back into the disordered flat phase. Possible applications of the model to the surfaces of ionic crystals are briefly discussed.
\end{abstract}

Keywords: Equilibrium thermodynamics and statistical mechanics; Ionic crystals; Ising models; Low index single crystal surfaces; Surface roughening; Surface thermodynamics

Solid-on-solid models play an important role in the description of phase transitions in crystal surfaces: they are realistic, yet simple enough to provide a deep insight into the physics of the disordering processes (deconstructions as well as roughening) which take place on surfaces.

In particular, the bcc(001) surface can be described by the BCSOS (body centered solid on solid) model, as follows. The bcc(001) surface is depicted in Fig. 1. It consists of two compenetrating square sublattices of atoms, shown as "black" (B) and "white" (W). The allowed levels $h_{i}^{\mathrm{B}}, h_{k}^{\mathrm{W}}$ of the surface atoms on the black and the white sublattice ${ }^{1}$ are even respectively odd multiples of $d=a / 2$, with $a$ the lattice spacing of either sublattice. In addition

\footnotetext{
* Corresponding author. Fax: +39 10 311066; e-mail: mazzeo@vaxgea.ge.infn.it.

${ }^{1}$ Crystallographically, if the black and white atoms are different the crystal structure is simple cubic.
}

two neighbouring $\mathrm{B}$ and $\mathrm{W}$ surface atoms are constrained to have height difference \pm 1 . This enables one to map the BCSOS model onto the six vertex

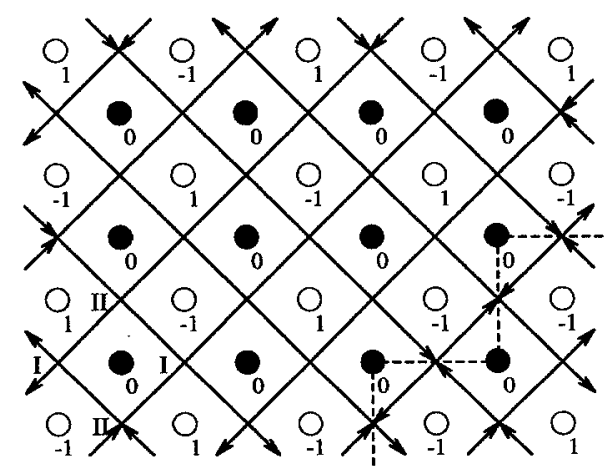

Fig. 1. The surface in its ground state seen from above, with the black and white atomic sublattices. In the lower right comer, an Ising wall is shown. The integers denote the values of the surface atomic heights in units of $d$. Arrows are drawn in accordance with Van Beijeren's construction [1]. The vertex lattice is divided into two sublattices, I and II. 
model [1], one of the few exactly solved models in 2D statistical mechanics. Arrows on the bonds of the dual lattice are drawn in such a way that always the higher of the two surface atoms separated by a given arrow is to the right of that arrow. The ice rule, i.e. the restriction to those vertices having two arrows pointing in and two out of a nodal point on the dual lattice, arises naturally from the uniqueness of the height variables on the surface (see Fig. 1).

Vertices $1,2,3$ and 4 describe local configurations where two neighbouring $\mathrm{B}$ (or W) atoms are found at different height values: in the sequel this situation will be referred to as a broken bond. The distinction between the different atomic species is reflected in the staggering of the vertex energies on two vertex sublattices, I and II. On sublattice I (see Figs. 1 and 2) vertices 1 and 2 correspond to a broken W-W bond and vertices 3 and 4 to a broken B-B bond. On sublattice II this situation is reversed. Vertices 5 and 6 describe surface elements without broken bonds: a system composed of vertices 5 and 6 only represents a microscopically flat surface. The energies to be assigned to a broken $\mathrm{W}-\mathrm{W}$ or $\mathrm{B}-\mathrm{B}$ bond will be $\epsilon, \epsilon^{\prime}=\epsilon+2 \delta$ respectively. Zero energy is assigned to vertices 5 and 6 . The corresponding Hamiltonian reads

$H=\frac{\epsilon}{2} \sum_{\langle i j\rangle}\left|h_{i}^{\mathrm{W}}-h_{j}^{\mathrm{W}}\right|+\frac{\epsilon^{\prime}}{2} \sum_{\langle k l\rangle}\left|h_{k}^{\mathrm{B}}-h_{l}^{\mathrm{B}}\right|$,

where the sums are restricted to neighbouring surface atoms of the same kind.

The model described by Eq. (1) has an exact solution for $\delta=0$ only (which corresponds to a single component crystal). This case is a well known prototype for a Kosterlitz-Thouless roughening transition of the surface, which takes place at $\beta \epsilon=\ln (2)$ $\left(\beta=1 / k_{\mathrm{B}} T, \quad k_{\mathrm{B}}=\right.$ Boltzmann's constant, $T=$ temperature).

The model for $\delta \neq 0$ has been previously introduced and studied by Knops [2], but only when both

\begin{tabular}{|c|c|c|c|c|c|}
\hline$\stackrel{0 \dagger_{1}}{\longrightarrow}$ & 21 & $\stackrel{1}{\rightarrow}$ & $1+2$ & of 1 & $1 / 0$ \\
\hline $\overrightarrow{x \mid 2}$ & $1 \sqrt{0}$ & $\overrightarrow{2} \backslash \overrightarrow{1}$ & $0 / 1$ & 170 & \begin{tabular}{l|l}
0 & 1
\end{tabular} \\
\hline 1 & 2 & 3 & 4 & 5 & 6 \\
\hline$\stackrel{\epsilon}{\epsilon+2 \delta}$ & $\stackrel{\epsilon}{\epsilon+2 \delta}$ & $\epsilon+2 \delta$ & $\epsilon+2 \delta$ & 0 & 0 \\
\hline
\end{tabular}

Fig. 2. The six vertices, the corresponding height values, and their energies for the two vertex sublattices.

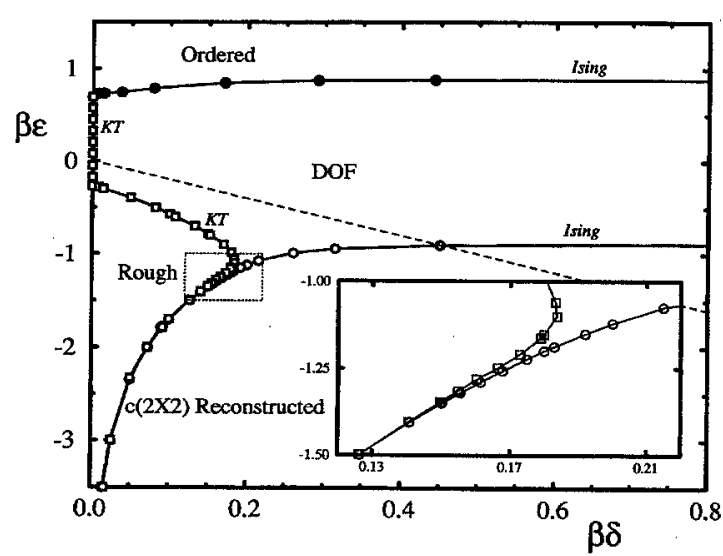

Fig. 3. The phase diagram. The dashed line is the line $\epsilon^{\prime}=\epsilon+2 \delta$ $=0$, below which the model may be applicable to ionic crystals. In the inset, an enlargement of the region where the Ising and the roughening curves approach each other is shown.

$\epsilon, \epsilon^{\prime}>0$. For $\delta>0$ (the $\delta<0$ case is easily obtained from this) he finds that the Kosterlitz-Thouless roughening transition at $\delta=0$ is replaced by a second order Ising disordering transition, and that roughening is pushed up to infinite temperature.

The case where both $\epsilon, \epsilon^{\prime}<0$, instead, is particularly suited to the description of two component systems with nearest neighbour B-W attraction (which is actually infinite in the BCSOS model) and next nearest neighbour repulsion. This model may be applicable to surfaces of ionic crystals, such as $\mathrm{CsCl}$, even though in reality further range interactions will exist, which have been neglected in the present model. The full phase diagram, which reveals a rich structure (see Fig. 3), has been completed only recently [3]. The techniques employed are the transfer matrix method (exact calculation of the partition function for a strip of $N \times \infty$ sites), finite size scaling analysis according to phenomenological renormalization group theory [4] and conformal field theory.

The surface has a $c(2 \times 2)$ reconstructed ground state, as shown in Fig. 1. Atoms in the less repulsive sublattice (B) are found at the same height level, atoms in the more strongly repulsive sublattice (W) alternate regularly up and down (to minimize energy the system has to break up as many bonds between $\mathrm{W}$ atoms as possible, which implies that no bonds between $\mathrm{B}$ atoms are broken). In terms of vertices, 
the ground state is formed by a network of straight paths of arrows, the vertical ones running alternatively upwards and downwards, the horizontal ones alternatively to the right and to the left, if Fig. 1 is rotated by $45^{\circ}$. As temperature is raised two different scenarios may open. If $\epsilon+2 \delta>0$ the surface undergoes an Ising disordering transition, and deconstructs into a kind of disordered flat (DOF) phase [5]. This can be easily understood in the limit $\delta \rightarrow \infty$. Here in fact the $B$ sublattice is infinitely strong, i.e. no broken bonds can occur between $\mathrm{B}$ atoms at any temperature. The $\mathrm{W}$ atoms are bound to lie just one vertical spacing either up or down with respect to the $B$ atoms, and the resulting configurations can be mapped exactly onto an antiferromagnetic Ising model. We notice that the system enters the same DOF phase reached after the disordering transition in the $\epsilon>0$ case, so that no other phase transition is found in crossing the $\epsilon=0$ line.

If $\epsilon+2 \delta<0$ instead, the Ising line is still present, but (for larger values of $|\epsilon| / \delta$ ) meets a Kosterlitz-Thouless roughening line, forming the boundary of a critical region inside which the surface is rough. The two lines seem to merge into a single one but they are just approaching each other exponentially fast. The actual non-coincidence of these two lines is not only obtained by numerical calculations, but is confirmed by exact arguments [6]. However, a crossing of the two lines is predicted if one naively considers the energy cost of the main kinds of defects on the surface related to deconstruction and roughening, i.e. Ising domain walls and steps, shown in Fig. 4. A domain wall divides two different Ising phases, and approximately costs an energy $-\epsilon=|\epsilon|$ per unit length with respect to the ground state energy. In terms of vertices it is composed of a connected sequence of vertices 5 and 6 . A step instead costs roughly $2 \delta$ per unit length; it consists of a connected path of arrows that are overturned with respect to the ground state configuration (notice that the step is actually a double step: due to the inequivalence of the two atomic sublattices it is energetically most favourable for the surface to break bonds always in the $\mathrm{W}$ sublattice, so that on both sides of the step the top surface layer is made of $\mathrm{W}$ atoms). Steps and domain walls are two a-priori not interfering objects: the same Ising phase is found at both sides of a step, as well as the surface is found at a)

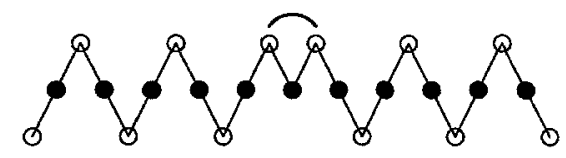

b)

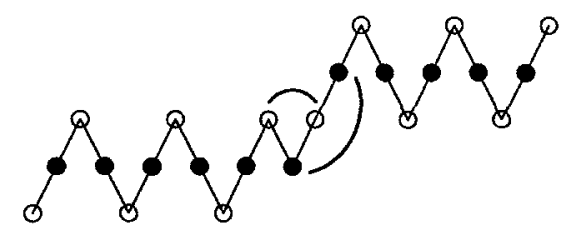

Fig. 4. Side view of an Ising domain wall (a) and of a double step (b) superimposed on the surface in its ground state. In (a) a broken bond between $\mathrm{W}$ atoms is missing: this amounts to an energetic cost of $-\epsilon=|\epsilon|$. In (b), in addition, a broken bond between $B$ atoms is present: the energetic cost is $-\epsilon+\epsilon^{\prime}=2 \delta$.

the same average height on both sides of a wall. If $|\epsilon| \ll \delta$ one could thus expect at high temperature proliferation on the surface of walls rather than steps, the opposite taking place if instead $|\epsilon| \gg \delta$. The first prediction is confirmed by the deconstruction undergone by the system, whereas the second scenario is never found: the phase diagram shows no roughening transition at temperatures lower than the Ising one, though this can be obtained by introducing further interactions between steps [6]. What happens instead is that a step locally, i.e. right along its path, destroys the Ising order, since, due to its structure, it overturns arrows with respect to the ground state. Below the roughening transition closed steps of finite total length are already present on the surface (not yet infinite steps since their free energy per unit length is still strictly positive). If they cover half of the surface, they manage to deconstruct it, leaving a flat surface which will roughen only at a slightly higher temperature. Investigations are still under way to clarify the universality class of the deconstruction transition, since critical exponents measured along the deconstruction line are seen to clearly deviate, for $\epsilon^{\prime}<0$, from the Ising values found in the $\epsilon^{\prime}>0$ region.

Another quite interesting result, though not completely unexpected [7], is the so called inverse roughening transition. The rough phase, lost at $\epsilon>0$ for $\delta \neq 0$ is recovered, as seen above, for $\beta \epsilon$ $<\frac{1}{2} \ln [2(1-\cos (\pi / 4))] \approx-0.2674$. This value follows from the exact solution of the non-staggered six 
vertex model $(\delta=0)$, and its determination proceeds from the study of the height-height correlation function in presence of steps of minimal height of two vertical lattice units. Once the surface has entered this phase, however, it will not remain rough at all temperatures, and will undergo another KosterlitzThouless phase transition back towards the DOF phase: at high temperature a rough surface will flatten again. Inverse roughening is an entropy driven phase transition, i.e. the system minimizes its free energy at high temperature by predominantly maximizing entropy rather than minimizing energy. Vertices 5 and 6 , which are of course not predominant when $\beta \epsilon$ is large and negative, start proliferating on the surface when $\beta|\epsilon|$ decreases. The increased entropy due to the larger number of configurations which can be formed with all the six vertices overcomes, in the free energy balance, the global energetic cost. For certain choices of the parameters a connected network of 5 and 6 vertices may be created, with a flattening effect on the surface, with vertices $1,2,3$ and 4 occupying the patches in between. However, it looks like in most cases this percolating network is completed by patches of the ground state, with B atoms at the same level. In either case the surface is not rough anymore, but is found in a DOF state.

In conclusion, a statistical mechanical model formulated by Knops has been extended and applied to the (001) surface of two component bcc crystals with nearest neighbour attractions and next nearest neighbour repulsions. We propose the model may find realizations in ionic crystals of $\mathrm{CsCl}$ type, where ions of the same type repel each other. Indeed the equilibrium shape of the crystal described by this model shows the typical behaviour experimentally found in ionic [8] crystals. To our knowledge, however, surface critical properties of such systems have not been investigated experimentally yet. In ionic systems the Coulombic interaction extends to further than next nearest neighbours, although it is screened at larger distances. We cannot presently tell whether these extended interactions will drastically modify the critical properties. It is our hope that our work will stimulate discussions and proposals for an experimental verification of some of the predicted results.

\section{References}

[1] H. van Beijeren, Phys. Rev. Lett. 38 (1977) 993.

[2] H.J.F. Knops, Phys. Rev. B 20 (1979) 4670

[3] G. Mazzeo, E. Carlon and H. van Beijeren, Phys. Rev. Lett. 74 (1995) 1391.

[4] P. Nightingale, J. Appl. Phys. 53 (1982) 7927.

[5] M. den Nijs, Phys. Rev B 46 (1992) 10386, and references therein.

[6] E. Carlon, G. Mazzeo and H. van Beijeren, in preparation.

[7] E. Luijten, H. van Beijeren and H.W.J. Blöte, Phys. Rev. Lett. 73 (1994) 456.

[8] E. Carlon, H. van Beijeren and G. Mazzeo, Phys. Rev. E, submitted. 